\title{
Designing Biopolymer Microthreads for Tissue Engineering and Regenerative Medicine
}

\author{
Megan P. O’Brien ${ }^{1} \cdot$ Meagan E. Carnes ${ }^{1} \cdot$ Raymond L. Page $^{1}$ • \\ Glenn R. Gaudette ${ }^{1}$ - George D. Pins ${ }^{1,2}$
}

Published online: 15 April 2016

(C) Springer International Publishing AG 2016

\begin{abstract}
Native tissue structures possess elaborate extracellular matrix $(\mathrm{ECM})$ architectures that inspire the design of fibrous structures in the field of regenerative medicine. We review the literature with respect to the successes and failures, as well as the future promise of biopolymer microthreads as scaffolds to promote endogenous and exogenous tissue regeneration. Biomimetic microthread tissue constructs have been proposed for the functional regeneration of tendon, ligament, skeletal muscle, and ventricular myocardial tissues. To date, biopolymer microthreads have demonstrated promising results as materials to recapitulate the hierarchical structure of simple and complex tissues and well as biochemical signaling cues to direct cell-mediated tissue regeneration. Biopolymer
\end{abstract}

This article is part of the Topical Collection on Artificial Tissues

Megan P. O'Brien and Meagan E. Carnes contributed equally to this work.

George D. Pins

gpins@wpi.edu

Megan P. O’Brien

mobrien2@wpi.edu

Meagan E. Carnes

mecarnes@wpi.edu

Raymond L. Page

rpage@wpi.edu

Glenn R. Gaudette

cgaudette@wpi.edu

1 Department of Biomedical Engineering, Worcester Polytechnic Institute, 100 Institute Rd, Worcester, MA 01609, USA

2 Department of Biomedical Engineering, Worcester Polytechnic Institute, Life Sciences and Bioengineering Center, Gateway Park, rm 4010, 100 Institute Road, Worcester, MA 01605-2615, USA microthreads have also demonstrated exciting potential as a platform technology for the targeted delivery of stem cells and therapeutic molecules. Future studies will focus on the design of microthread-based tissue analogs that strategically integrate growth factors and progenitor cells to temporally direct cellmediated processes that promote enhanced functional tissue regeneration.

Keywords Microthreads · Biopolymer · Targeted stem cell delivery $\cdot$ Musculoskeletal tissue regeneration $\cdot$ Cardiac tissue regeneration

\section{Introduction}

Tissue engineering is a multidisciplinary field that integrates the principles of engineering, material science, cell biology, and medical sciences to create scaffolds that promote regeneration of diseased or damaged tissues and organs. The primary functions of these scaffolds are to provide provisional structural and mechanical stability to the injured tissue. Additionally, these scaffolds should provide physical (topographic) and biochemical signaling cues that mimic native extracellular matrix (ECM) architecture to instruct progenitor cells to regenerate functional tissue.

In native tissue, the principle structural components of ECM are comprised of fibrillar elements, arranged into hierarchically ordered constructs. These fibrillar elements are composed of protein-based building blocks that form structures 20-200 nm in diameter and contain matrix proteins, such as type I collagen and fibrin. The structural arrangement of these fibrils varies from tissue to tissue, in a manner that optimizes their functional mechanical properties. The distinct architectures and biochemical compositions of the native 
tissues also serve a critical function in directing cell-mediated tissue regeneration following injury or trauma [1].

Biopolymer microthreads are discrete, fibrous materials, generated from ECM and naturally derived proteins such as fibrin $[2,3 \cdot 4]$, silk $[5,6]$, collagen $[7,8]$, chitosan, and alginate [9-11]. The morphological and biochemical properties of the these microthreads are comparable to native fibrous structures, and they can be precisely engineered into hierarchically ordered, tissue-specific scaffolds with morphological, mechanical, and biochemical cues to promote cell-mediated tissue regeneration (Fig. 1). Using a variety of processing strategies, including chemical cross-linking, these microthreads can be modified to precisely modulate their material properties including the tensile strengths and degradation rates. These thread modifications enable the fabrication of scaffolds that closely mimic properties of native tissues.

Biopolymer microthreads can be used as a biomaterials platform technology for numerous applications including the localized release of therapeutic molecules, growth factors, and stem cells. Designing microthreads containing growth factors with distinct release kinetics facilitates scaffold-mediated control of the temporal regenerative events that promote tissue repair. Incorporating cells, including pluripotent stem cells, into microthreads may overcome challenges of limited cellular ingrowth into wound sites. Stem cells are a powerful therapeutic strategy, with proliferative capacity and the potential to differentiate into a variety of cell types with different phenotypic functions $[12,13 \cdot 14]$. Microthreads have been used to deliver stem cells to a damaged tissue and may be able to direct cells down a desired lineage by integrating specific physical and biochemical cues into the scaffold $[4,15]$.

We anticipate that microthread-based scaffolds will enable an off-the-shelf, regenerative solution that will ultimately replace the current standard treatments for a wide variety of tissue types. Toward this, the fibrin microthread technology platform is being brought to the market by VitaThreads, LLC. It is anticipated that the first product, an acellular fibrin microthread suture to enhance aesthetic wound closure, will conclude preclinical testing within the next 18-24 months and begin its initial clinical trials immediately thereafter. Advances in cell therapy will likely parallel regulatory approval of fibrin microthreads, allowing for the development of many exciting combination products, such as the therapies discussed in the following sections.

In this review, we will explore innovative approaches for creating biopolymer microthread-based scaffolds that integrate morphologic, mechanical, and biochemical cues of native tissues to promote functional tissue regeneration (Table 1). Progress that has been made toward the development of microthread-based scaffolds for use in treating tendon, ligament, and skeletal muscle injury and state-of-the art studies that promise to resolve the ongoing challenges

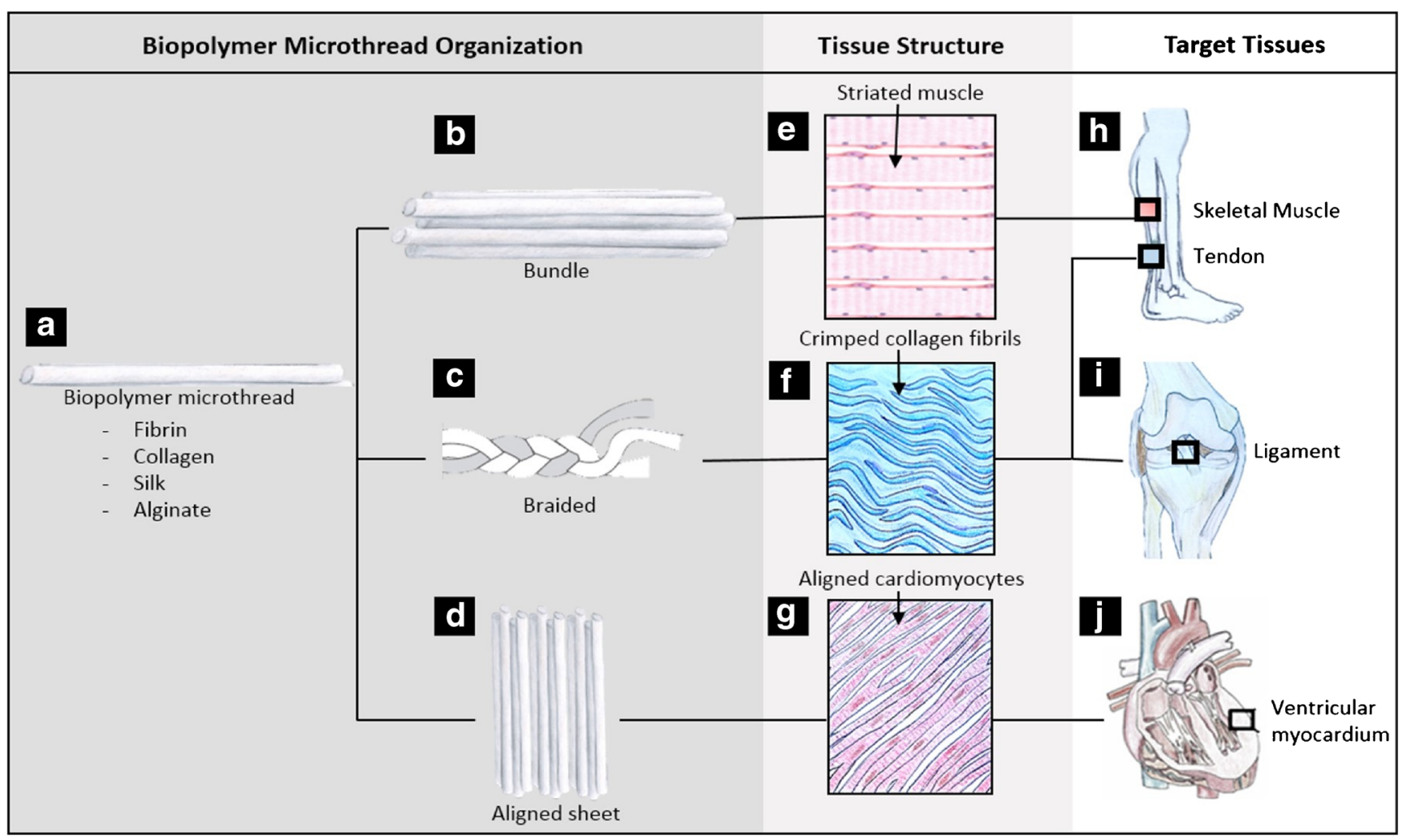

Fig. 1 Biopolymer microthreads composed of different biomaterials (a) can be hierarchically combined to produce scaffolds (b-d). These scaffolds mimic tissue structures $(\mathbf{e}-\mathbf{g})$ of targeted diseased or injured tissues $(\mathbf{h}-\mathbf{j})$ 
Table 1 Biopolymer microthreads made of varying materials have been strategically combined and modified to form structured scaffolds that mimic targeted tissues

\begin{tabular}{|c|c|c|c|c|}
\hline Material & Targeted tissue & Structures & Goal of work & Reference \\
\hline \multirow[t]{4}{*}{ Fibrin } & Ventricular myocardium & Suture (bundled fibrin microthreads) & $\begin{array}{l}\text { In vivo, cell delivery (hMSCs) } \\
\text { In vitro, cellular response ( } \mathrm{hMSCs} \text { ) } \\
\text { In vivo, cell delivery (hMSCs) }\end{array}$ & $\begin{array}{l}\text { Guyette et al. } 2013[3 \bullet] \\
\text { Proulx et al. } 2011[16] \\
\text { Tao et al. } 2014 \text { [17] }\end{array}$ \\
\hline & Skeletal muscle & Microthreads & $\begin{array}{l}\text { Mechanical modification } \\
\text { Mechanical modification } \\
\text { In vivo, GF incorporation } \\
\text { In vivo, cell delivery } \\
\quad \text { (primary human satellite cells) }\end{array}$ & $\begin{array}{l}\text { Grasman et al. } 2012[18] \\
\text { Grasman et al. } 2014[2] \\
\text { Grasman et al. } 2015[19 \bullet] \\
\text { Page et al. } 2011[4]\end{array}$ \\
\hline & \multirow[t]{2}{*}{ Tendon/ligament } & & $\begin{array}{l}\text { In vitro cellular response, } \\
\text { mechanical modification }\end{array}$ & Cornwell et al. 2007 [20] \\
\hline & & & In vitro, GF delivery & Cornwell et al. 2010 [21] \\
\hline \multirow[t]{3}{*}{ Silk } & \multirow[t]{3}{*}{ Tendon/ligament } & Bundles & $\begin{array}{l}\text { In vitro cellular response, } \\
\text { mechanical modification }\end{array}$ & Altman et al. 2002 [6] \\
\hline & & Knitted & In vitro cellular response & Liu et al. 2008 [15] \\
\hline & & Braided, twisted, cabled & $\begin{array}{l}\text { Mechanical modification } \\
\text { In vitro cellular response }\end{array}$ & $\begin{array}{l}\text { Horan et al. [22] } \\
\text { Chen et al. } 2003 \text { [23] }\end{array}$ \\
\hline \multirow[t]{9}{*}{ Collagen } & \multirow[t]{9}{*}{ Tendon/ligament } & \multirow[t]{7}{*}{ Microthreads } & $\begin{array}{l}\text { In vitro cellular response, } \\
\text { mechanical modification }\end{array}$ & Cornwell et al. 2007 [7] \\
\hline & & & Mechanical modification & Dunn et al. 1992 [24] \\
\hline & & & Mechanical modification & Zeugolis et al. 2009 [25] \\
\hline & & & Mechanical modification & Pins et al. 1997 [26] \\
\hline & & & Mechanical modification & Zeugolis et al. 2010 [27] \\
\hline & & & $\begin{array}{l}\text { In vitro cellular response, } \\
\text { mechanical modification }\end{array}$ & Gentleman et al. 2003 [8] \\
\hline & & & In vivo, mechanical modification & Kato et al. 1991 [28] \\
\hline & & Braided & $\begin{array}{l}\text { In vitro cellular response, } \\
\text { mechanical modification }\end{array}$ & Walters et al. 2012 [29] \\
\hline & & Composite (fibers and gel) & In vivo, cell delivery (BMSCs) & Awad et al. 2003 [30] \\
\hline \multirow[t]{2}{*}{ Chitosan/hyluronan } & \multirow[t]{2}{*}{ Tendon/ligament } & \multirow[t]{2}{*}{ Braided composite fibers } & In vivo, cell delivery (fibroblasts) & Funakoshi et al. 2005 [9] \\
\hline & & & In vitro cellular response & Funakoshi et al. 2005 [10] \\
\hline
\end{tabular}

GF growth factor, hMSCs human mesenchymal stem cells, MSCs mesenchymal stem cells, BMSCs bone marrow derived mesenchymal stem cells

associated with these strategies are reviewed. A new strategy for developing microthread-based scaffolds as devices to facilitate targeted delivery of therapeutic molecules, growth factors, or stem cells to damaged tissue is also discussed. Finally, strategies for creating anisotropically aligned, composite scaffolds to regenerate complex tissues such as ventricular myocardial tissue are suggested.

\section{Tendon and Ligament}

\section{Regenerative Need}

Torn tendons and ligaments are among the most common sports injuries, with over 200,000 patients per year injuring their anterior cruciate ligament (ACL) alone [31]. If ACL damage is left untreated, it can result in arthritis, joint laxity, and chronic pain. Current treatments for ACL injuries include autografts, allografts, and xenografts. The "gold standard" treatment for ACL injuries involves replacing the damaged ACL with a patellar tendon autograft, but this procedure can result in donor site morbidity, fracture, and tendonitis. Similarly, allografts and xenografts have been used for ACL repair, but these treatments present a risk of immune rejection and disease transmission and they can take longer to heal than autografts [32•]. As such, there is a need for an off-the-shelf tendon/ligament replacement that is biocompatible, provides mechanical support to the joint, and facilitates long-term joint stability.

\section{Scaffold Design Strategies}

Tendons and ligaments are composed primarily of bundles of type I collagen fibers, organized in a dense and highly aligned, cable-like manner with a crimp pattern that repeats every 45 $60 \mu \mathrm{m}$ [33]. These fiber bundles form fascicles within the cable-like structure that are principally responsible for uniaxial load transfer from muscle to bone (tendon) or bone to bone (ligament). Ultimate load of the ACL was determined to be $2,160 \pm 157 \mathrm{~N}$ for specimens aged 22 to 35 [34]. To promote robust tendon/ligament regeneration, these biopolymer scaffolds should provide provisional tensile strengths and strains, 
recapitulate the complex tissue architecture and ECM composition, and present biochemical signaling cues to direct cellmediated tissue regeneration of functional tissue constructs.

Many researchers have investigated developing novel cross-linking strategies to improve the tensile strengths and stiffnesses of discrete collagen microthreads to enhance their structural stability and to prolong their degradation times $[7,8$, 24-28]. Initial studies of discrete, reconstituted collagen fibers used combinations of dehydrothermal treatment (DHT), carbodiimide, and glutaraldehyde cross-linking methods to create scaffolds with tensile strengths, stiffnesses, and degradation rates that would maximize neotendon and neoligament regeneration $[24,28]$ When these scaffolds were implanted in a rabbit Achilles tendon model for 52 weeks, histological analyses showed that glutaraldehyde-cross-linked collagen fiber implants were not resorbed and triggered a fibrotic encapsulation response. Carbodiimide-cross-linked collagen fibers were resorbed and replaced with neotendon, but lacked mechanical properties that were comparable to native tendon [28]. The results of these studies suggested that the next generation of collagen microthread scaffolds exhibits tensile strengths comparable to native tendon and slower degradation rates to enhance the rate of aligned tissue deposition on the surfaces of the scaffolds. To further analyze the mechanical properties, enzymatic degradation rates, and fibroblast outgrowth on collagen microthreads, one study cross-linked threads using physical and chemical cross-linking techniques including dehydrothermal treatment (DHT), ultraviolet light (UV), and carbodiimide (EDC) cross-linking [7]. They found that DHT cross-linked collagen microthreads had tensile strengths comparable to native tendon fibers and prolonged the degradation rates. However, these cross-linked collagen threads also decreased the rate of fibroblast outgrowth on the surfaces of the threads in an in vitro tissue ingrowth assay [7]. These findings suggest that the design of future microthreads must incorporate biochemical signaling cues to enhance the rate of cellular ingrowth and tissue regeneration.

To create a scaffold with biochemical signaling cues to enhance the rate of cell-mediated tissue regeneration, we developed a novel process for biofabricating fibrin microthreads, derived from the provisional ECM protein that triggers the initial cellular responses to wound healing [20]. When fibrin microthreads were chemically or physically cross-linked using methods described previously, they exhibited structural, mechanical, and cell attachment properties similar to collagen microthreads [20]. These results suggest that fibrin microthreads are an innovative platform technology for directing cell-mediated tissue regeneration. Several examples of promising results for fibrin microthread-based scaffolds are illustrated later in this review.

To create scaffolds that more closely replicate the complex structural and mechanical properties of tendons and ligaments, biopolymer microthreads have been engineered into tissue constructs with braided, twisted, or bundled morphologies. This provides a strategy to increase mechanical strength while simultaneously mimicking native ECM architecture. In one study, EDC cross-linked braided collagen scaffolds were developed with mechanical properties matching native ligament, and they also supported increased primary rat ligament fibroblast proliferation [29]. In a systematic series of studies, silk fibers were formed into plied, twisted, cabled, braided, and textured geometries, and it was shown that cabled silk fibers closely match native tissue mechanical properties including ultimate tensile strength and stiffness, as well as fatigue performance $[6,22]$. Additionally, Altman et al. showed that these scaffolds supported human progenitor bone marrow stem cell attachment, proliferation, and differentiation, as well as increased messenger RNA (mRNA) expression of ligament markers such as collagen types I and III [6]. Finally, a composite scaffold composed of knitted silk fibers encapsulated with a collagen sponge, combining the mechanical strength and slow degradation of silk with the increased expression of ligament matrix genes of cells cultured on collagen substrates. When implanted in a rabbit MCL defect model, the silk/collagen scaffold had better collagen fiber deposition and stronger scaffold ligament interface than untreated controls and controls treated with silk scaffolds [23]. Together, these findings suggest that biopolymer microthreads provide a platform for creating more complex scaffold geometries, capable of recapitulating native tissue architecture and mechanical properties to promote cell-mediated, functional tissue regeneration.

To further enhance cellular response, researchers have focused on incorporating biochemical signaling cues into biopolymer microthreads to enhance cellular and tissue responses to the scaffolds. In one study, fibrin microthreads loaded with varied concentrations of FGF-2 were incorporated into an in vitro model of tissue ingrowth and the scaffolds promoted an increase in human dermal fibroblast proliferation and migration on the surfaces of materials when compared to controls [21]. When the ECM peptide sequence arginine-glycineaspartic acid (RGD) was incorporated into silk fibers, the RGD-modified silk fibers increased collagen type I transcript levels and improved attachment of bone marrow stromal cells and ACL fibroblasts [23]. Additionally, human tenocytes had a 1.3-fold increase in attachment and 2.3-fold increase in type I collagen mRNA levels when seeded on RGD-modified silk sutures, compared to cells seeded on tissue culture plastic [35]. These findings suggest that biochemical modifications are a promising strategy for eliciting cellular response and enhancing the rate to functional tissue regeneration in implantation models.

Biopolymer microthreads have also been used as a cellular delivery mechanism to enhance the rate of tissue regeneration in models of tendon and ligament repair. Delivery of stem cells to an injured tendon or ligament aids in tissue 
regeneration and functional recovery [36, 37]. Awad et al. seeded rabbit bone marrow-derived mesenchymal stem cells (BMSCs) onto collagen gel/fiber composite scaffolds that were used to replace patellar tendon defects created in a rabbit animal model [30]. After 26 weeks, the maximum force, stiffness, and strain of MSC-seeded grafted repairs were 174, 183, and $192 \%$ greater than values for ungrafted contralateral injury controls, respectively [30]. Liu et al. performed a study comparing BMSCs and ACL fibroblasts to determine the most effective cell type for treating tendon injury [15]. Both cell types were cultured on silk scaffolds and implanted into a rabbit ACL injury model. They found that a greater number of BMSCs were localized at the injury site than ACL fibroblasts, suggesting that BMSCs are a better cell type for treating tendon injury [15]. Funakoshi et al. developed a braided scaffold of chitosan/hyluronan polymer fibers and tested these fibers as a potential tendon/ligament treatment in a rabbit rotator cuff model [9]. They found that type I collagen was formed only in the fibroblast-seeded scaffold and the tensile strength of this scaffold was significantly enhanced 12 weeks after surgery compared to untreated control injuries [9]. Biopolymer microthreads support enhanced cellular responses such as attachment and proliferation, and when implanted have been shown to enhance tissue regeneration.

\section{Limitations and Future Needs}

Biopolymer microthreads are a promising approach to tendon/ ligament tissue engineering, as they mimic the native ECM architecture. Significant work has been done to braid, twist, or cable biopolymer microthreads to form robust, mechanically supportive scaffolds suitable for tendon/ligament repair. Although these scaffolds have adequate mechanical properties, there is still a need to create a scaffold with scaffold degradation rates that promote sufficient tissue ingrowth.

One promising approach to enhancing the rate of tendon/ ligament regeneration is to create composite scaffolds that integrate the benefits of several materials and cell signaling cues. For example, researchers created a composite silk fiber/ collagen sponge scaffold, which increased the expression of tendon/ligament genes, compared to a silk fiber scaffold, in a murine implantation model [38]. Microthread-based tissue analogs that combine the mechanical and biochemical signaling cues of collagen and fibrin threads may also enhance the rate on tissue regeneration.

The controlled release of growth factors from microthreads is another promising strategy to promote tendon/ligament regeneration. Tendon and ligament regeneration is controlled by a variety of growth factors, including insulin-like growth factor-I (IGF-I), transforming growth factor- $\beta$ (TGF- $\beta$ ), vascular endothelial growth factor (VEGF), platelet-derived growth factor (PDGF), and basic fibroblastic growth factor (bFGF) [39]. In the future, dose responses, temporal release profiles, and combinatorial effects should be studied to investigate how growth factors will promote in vivo tendon/ ligament regeneration. Currently, the only commercially available scaffolds on the market for tendon and ligament repair are allograft scaffolds. Biopolymer microthread-based sutures for wound closure are currently undergoing preclinical testing, and it is anticipated that subsequent evaluation in clinical trials will conclude within the next 2-3 years. At the conclusion of these trials, we anticipate that fibrin microthreads augmented with growth factors and/or cytokines with controlled release profiles that promote tendon and ligament regeneration would begin preclinical and clinical assessment to assess the safety and efficacy of these implantable scaffolds.

\section{Skeletal Muscle}

\section{Regenerative Need}

Combat injuries, tumor resection, and traumatic injury can result in volumetric muscle loss (VML), where severe musculoskeletal damage results in scar formation and poor functional tissue recovery. For all soldiers injured on the battlefield, $82 \%$ suffer from at least one musculoskeletal extremity wound [40]. Following these injuries, endogenous skeletal muscle regeneration activates muscle progenitor cells or satellite cells, which proliferate and differentiate to form mature myotubes that restore contractile function to damaged muscle tissue [41]. In the case of VML injuries, the structural and biochemical cues such as the basement membrane and connective tissue are removed, compromising regeneration potential of the tissue. The current standard of care for VML is autologous tissue transfer from an uninjured site. This procedure often results in donor site morbidity, loss of muscle strength due to the formation of scar tissue, and graft failure from complications such as infection and necrosis [42-45]. As such, there is a need for an off-the-shelf biomimetic scaffold that directs the formation of new skeletal muscle tissue in large defect sites and enhances functional tissue regeneration.

\section{Scaffold Design Strategy}

Skeletal muscle is a highly aligned tissue, which represents approximately $40-45 \%$ of total body mass [46], and it is primarily responsible for generating a series of discrete uniaxial forces that enables locomotion. Skeletal muscle is composed of nerves, blood vessels, connective tissue, and myofibers, the fibrous functional units of muscle. Myofibers form when myoblasts fuse together and form multinucleated tubes, ranging in size between 20 and $100 \mu \mathrm{m}$ [46]. Myofibers are surrounded by connective tissue comprised of ECM proteins, which provides structural support and aids in synchronous contraction. Skeletal muscle has a stiffness ranging from 
$12 \mathrm{kPa}$ to $11.2 \mathrm{MPa}$ depending on whether it is static or contracting $[47,48]$. Scaffolds that direct functional skeletal muscle regeneration must provide structural and biochemical cues to guide tissue deposition from the surrounding musculature into large VML defects [49•, 50].

Scaffolds that guide skeletal muscle regeneration strategies must facilitate the formation of aligned myotubes parallel to the force conduction pathway, to maximize functional regeneration. Specifically, these tissue scaffolds must provide the morphological architecture, mechanical strengths, degradation rates, and cell signaling cues to promote cellular ingrowth [51]. Biopolymer scaffolds composed of ECM proteins such as collagen and fibrin contain intrinsic bioactive signaling cues to promote specific cellular functions, but few studies describe the development of scaffolds that provide both biochemical and structural cues to direct aligned, musculoskeletal tissue ingrowth [52•]. Fibrin microthreads offer a promising therapeutic treatment for skeletal muscle regeneration $[4,19 \bullet]$. These materials approximate the ECM of native skeletal muscle and serve as structural support with bioactive cues to guide myotube fusion and differentiation into contractile muscle.

To investigate the potential for fibrin microthreads to enhance skeletal muscle regeneration, cell-seeded threads were studied in a murine VML defect model system [4]. Primary human muscle cells expressing stem-cell markers were cultured on fibrin microthreads and then secured in an excised murine tibialis anterior (TA) muscle. Cell-seeded fibrin microthread treatment groups appeared to reduce collagen deposition and increased force production compared to untreated control injuries at later time points (4 months) [4]. Histological analyses indicated that implanted cells contributed to myotube formation and muscle regeneration. Further evaluation noted that microthreads were rapidly degraded within 2 weeks after implantation and many of the regenerated myofibers in the wound site exhibited misalignment with respect to the direction of the muscle tissue. These and other studies suggest that the persistence of the scaffold in the wound site is critical for directing aligned myofiber ingrowth and robust tissue regeneration $[4,53]$.

These findings motivated a series of in vitro studies to modulate the structural and mechanical properties of fibrin microthreads to enhance cellular responses and aligned tissue ingrowth. To strategically tune the mechanical properties and degradation rates of fibrin microthreads, researchers investigated various chemical and physical cross-linking strategies $[18,20]$. One study showed that physically cross-linking threads with ultraviolet (UV) irradiation increase mechanical strength and stiffness of fibrin microthreads twofold [20]. In a similar study, fibrin microthreads were cross-linked with 1ethyl-3-(3-dimethylaminopropyl) carbodiimide (EDC) in a dose-dependent manner, as a function of $\mathrm{pH}$ and time. The results of this study demonstrated that cross-linking threads with EDC in a neutral buffer (EDCn) significantly increased the tensile strength and decreased the degradation rates of the materials sixfold, relative to uncross-linked control threads, without adversely effecting cell attachment or proliferation [18]. Together, these findings suggest that cross-linking fibrin microthreads will enable their persistence in the wound, providing extended structural support to large-scale injuries and enhancing aligned myofiber ingrowth.

In a study investigating an alternative method to modulate the structural and mechanical properties of fibrin microthreads, scaffolds were subjected to a series of static axial stretching conditions [2]. When microthreads were stretched between 0 and $200 \%$ of their initial lengths, threads stretched $150 \%$ of their initial length exhibited a threefold increase in tensile strength [2]. Additionally, stretched threads increased the alignment of $\mathrm{C} 2 \mathrm{C} 12$ myoblasts seeded on the surface of threads, with respect to untreated microthread controls [2]. Together, these findings show that static axial stretching significantly enhanced mechanical properties and the cellular responses of fibrin microthreads. However, static axial stretching did not modulate the in vitro degradation rates of fibrin microthreads, implying that future work should address this in order to maximize their regenerative capacity.

Growth factors serve an important role in modulating the temporal sequence of processes that direct functional skeletal muscle regeneration. As such, a key consideration for the design of microthread-based scaffolds is the controlled release of therapeutic molecules in a manner that recapitulates the complex signaling cues during tissue regeneration, similar to previous studies on the controlled release of FGF-2 [21]. During muscle wound healing, hepatocyte growth factor (HGF) stimulates the activation and migration of satellite cells (SCs) from the wound margin to the injury site [54, 55]. When HGF was adsorbed to the surface of uncross-linked and EDCn cross-linked fibrin microthreads, functional analyses of HGF release, using an in vitro cell proliferation assay, showed that EDCn cross-linked microthreads facilitated a rapid release of HGF from the microthreads for a period of 48-72 $\mathrm{h}$ [56]. These results suggest that fibrin microthreads with precisely tuned degradation rates and growth factor release kinetics can enhance the functional regeneration of skeletal muscle tissue.

To investigate the effects of cross-linking and growth factor release from microthreads on skeletal muscle regeneration in an in vivo model, fibrin microthreads that were EDCn crosslinked and loaded with HGF were implanted in a murine model of a VML defect where $20 \%$ of the TA muscle was excised [19•]. HGF loaded, EDCn cross-linked threads increased the number of differentiated myoblasts 14 days after injury and supported enhanced angiogenesis, relative to control threads [19•]. The rapid, sustained release of HGF from the microthreads also significantly enhanced the force production of muscle tissue 60 days after injury, compared to untreated controls [19•]. Enhanced force generation suggests that fibrin microthreads are a promising new therapeutic strategy for the functional recovery of VML injuries. 


\section{Limitations and Future Needs}

Biopolymer microthreads are a promising approach to skeletal muscle regeneration as they recapitulate the morphology of native skeletal muscle fibers and the surrounding ECM to provide topographical cues that promote aligned myotube formation. To achieve functional, organized tissue regeneration, modifications to enhance degradation rates are still needed.

With the development of more sophisticated scaffolds, skeletal muscle regeneration strategies should continue to incorporate therapeutic proteins to promote the endogenous regeneration of tissue by initiating the response of resident satellite cells. As biomaterial scaffolds advance, strategies to incorporate multiple growth factors with clinically relevant release profiles may be helpful in promoting various stages of tissue regeneration. These inductive scaffolds should release a sequence of growth factors that might promote resident progenitor cells to infiltrate the wound site $[57,58]$, proliferate $[54,59]$, and differentiate $[60,61]$ to form mature, functional myotubes.

Additional strategies for delivering satellite cells or stem cells to the injury site are still needed to accelerate the regeneration of VML injuries. Current strategies focus on hydrogelbased delivery, which provides limited functional support. Biopolymer microthreads used for cell delivery may integrate mechanical support, biomimetic tissue architecture, and topographical cues to enhance the formation of aligned, functional tissue. Currently, there are no commercially available scaffolds on the market to direct skeletal muscle repair. Challenges such as reinnervation and vascularization have limited the translation of tissue-engineered products for skeletal muscle [62]. At the conclusion of the clinical trials for fibrin microthread-based sutures, we anticipate that we can begin preclinical and clinical assessments of microthread scaffolds augmented with myogenic, angiogenic, and/or neurotrophic growth factors with controlled release profiles that promote endogenous skeletal muscle regeneration in small facial muscle defects and, subsequently, large VML defects.

\section{Ventricular Myocardium}

\section{Regenerative Need}

Heart failure is the leading cause of death globally with 500, 000 new cases diagnosed annually in the USA alone [63]. When a coronary artery is blocked, a myocardial infarction (MI) can occur resulting in downstream ischemia of cardiac muscle. The ischemia can lead to cardiomyocyte death and a severe inflammatory reaction resulting in non-contractile scar formation [64]. Decreased contractile function of the ventricle can ultimately lead to heart failure [65]. The current gold standard treatment is a heart transplant, but donors are limited.
Only $\sim 2500$ transplantation surgeries occur annually, leaving a high majority untreated [63]. Other options such as left ventricular assist devices provide mechanical assistance but do not help to regenerate the damaged tissue. Because cardiomyocytes have a low proliferation rate, surrounding myocardium has a limited capacity to regenerate damaged tissue post-MI. Consequently, there is a significant need for a regenerative strategy to restore the functional properties of damaged myocardial tissue. Two critical components to this strategy are the development of (i) engineered scaffolds and (ii) cell delivery approaches that will facilitate the regeneration of contractile myocardial tissue.

\section{Scaffold Design Strategy}

The anterior myocardium is $\sim 1.5 \mathrm{~cm}$ thick and composed of highly aligned cardiomyocytes and ECM fibrils, predominantly containing collagen [66, 67]. Ventricular myocardium is arranged in anisotropically aligned sheets of fibrils that are stacked and rotate from $+60^{\circ}$ to $-60^{\circ}$ [67]. Within each layer, the fibril size can range from 30 to $120 \mathrm{~nm}$ with fibril orientations ranging from $\pm 13^{\circ}$ relative to one another $[68,69]$. Myocytes closely follow this structure, resulting in highly oriented cells and aligned contraction of cells. This alignment results in high torsional squeezing enabling efficient pumping of blood [70]. Ventricular myocardium has a modulus ranging from 20 to $500 \mathrm{kPa}$ as it moves from diastole to systole [71••]. To promote functional tissue regeneration, recapitulating the properties of the native tissue may be advantageous [71••, 72].

Since native cardiomyocytes exhibit limited proliferative and regenerative capacity, significant research has focused on delivery of cells to the infarct site. Current methods include intravascular (IV), intracoronary (IC), and intramyocardial (IM) delivery. Intravascular delivery is the least invasive and least efficient, with a substantial number of cells trapped in the lungs [73], and less than $1 \%$ of the cells residing in the infarcted region [74]. The use of IC delivery enables cells to be delivered directly to the region of interest during angioplasty. However, when blood flow is restored, the majority of cells are washed away from the infarct with only $3 \%$ of the delivered cells engrafted into the heart $4 \mathrm{~h}$ after delivery [75]. The IM route for injection of cells improves retention, with $11 \%$ of the cells engrafting in the heart $1 \mathrm{~h}$ after delivery [75]. Scaffold-based strategies for delivering stem cells to the heart have also been investigated using materials such as alginate [76], collagen [77], collagen/GAG [78], and Matrigel [79, 80]. However, stem cells delivered via scaffolds have difficulty transversing the myocardial wall to reach the endocardium [77], where most clinical myocardial infarctions reside. Thus, current methods cannot efficiently deliver stem cells to a well-defined region of the heart such as a myocardial infarct.

To enhance the efficiency of cell delivery, our labs pioneered a cell-seeded biopolymer microthread-based suture 
system to facilitate targeted, localized cell delivery in the heart. Biopolymer threads may mimic the fibril architecture observed in native myocardium and may help cells to incorporate into the infarct tissue. In one study evaluating the effective delivery of human mesenchymal stem cells (hMSCs), cells were incorporated on a fibrin microthread suture and implanted or delivered via IM injection to the left ventricular wall of non-infarcted normal rat hearts. Delivery efficiency was greatly improved using the fibrin suture relative to the cell injection alone $(63.6 \pm 10.6$ and $11.8 \pm 6.2 \%$, respectively) [3•]. In a more recent study, an acellular suture was compared to an hMSC-seeded suture to assess the impact in normal functioning rat hearts. To compare regional mechanical function, systolic area of contraction (SAC) in the region of biopolymer microthread delivery was evaluated. Compared to native myocardium, SAC was decreased for acellular scaffold implants, while this decrease was less pronounced in the hMSC-seeded suture group. Fibrosis was reduced for the hMSC-seeded suture group relative to the acellular suture group [17], suggesting that hMSCs may release factors limiting the fibrotic response to injury. The incorporation of hMSCs improved regional mechanics when compared to an acellular scaffold, demonstrating the value of delivering healthy cells incorporated onto the biomaterial scaffold. In an in vitro analysis, fibrin microthread sutures were able to support hMSCs through maintained viability, proliferative capacity, and multipotency as evaluated through a LIVE/DEAD assay, Ki-67 staining, and assays to differentiate the cells into adipocytes or osteocytes. Strategic incorporation of cellspecific ECM cell adhesion proteins such as fibronectin [81] may increase stem cell retention and delivery capacity. These data suggest that the fibrin microthreads are a platform technology [16] and are adaptable to other applications.

\section{Limitations and Future Needs}

Microthread-based scaffolds offer distinct advantages over cellular injections including higher cell retention and precise delivery to target damaged myocardium. Cellular injections are currently in clinical trials [82] but have low cell engraftment rates limiting delivery of healthy cells to an infarct. Given the well-defined fiber orientation in the heart, microthreads may be combined to form a highly aligned scaffold. Despite significant advances in the design of scaffolds for cardiac tissue engineering, there remains a need to develop an implantable cardiac patch with structural integrity that promotes substantial functional improvements to damaged tissue. While creation of a fibrin microthread-based scaffold is still in the development stages, contraction of individual microthreads seeded with cardiac myocytes suggests that a contractile cardiac patch is a promising future direction.

While improvement in regional mechanics was observed in an hMSC-seeded construct relative to an acellular construct,
hMSCs are not the most clinically relevant cell type as they do not readily transdifferentiate into cardiomyocytes. These stem cells may help reduce infarct expansion through paracrine factors but will not contribute to the active systolic function of the heart [83]. Induced pluripotent stem cell (iPS) derived cardiomyocytes may be a more relevant cell type for regeneration of contractile function. Incorporation of iPS derived cardiomyocytes on a biopolymer scaffold may be able to restore contractile function to infarcted regions of the heart.

While fibrin microthreads have shown the ability to guide cellular orientation [2], the microthreads are mechanically stiff relative to myocardium and may limit cell-mediated contractility [84]. Similarly, electrospun scaffolds have been shown to influence cellular orientation through the microtopography of fibrils [85] but are typically dense, limiting cellular infiltration and high-force contractions [71••]. In contrast, hydrogels provide compactible environments and have produced some of the highest reported contractile force measurements to date [86]. However, hydrogels are typically mechanically weak $[87,88]$ and may fail in the dynamic environment of the heart $[72,88]$. A composite material composed of a microthread element and a hydrogel phase may yield a scaffold combining the benefits of structural cues and mechanical integrity of microthreads, with the hydrogel environment that is conducive to cell-mediated contractility $[89,90]$. These materials could be strategically combined to mimic the mechanical environment of native myocardium promoting high functionality of incorporated cells. The use of fibrin microthreads within the composite may enable a contractile tissue analog of the myocardial tissue.

\section{Conclusions}

Biopolymer microthreads are natural, biodegradable scaffolds that can be manipulated mechanically and biochemically to mimic tissue-specific structural properties. Complex tissue structures can be recapitulated with these scaffolds, providing the necessary cues to promote cell-mediated tissue regeneration. When incorporating growth factors, microthreads become a powerful tool to drive regeneration processes in vivo. Through the delivery of stem cells on microthreads, injured or diseased tissues can receive targeted treatment with a reduced dependence on tissue ingrowth. A wide range of applications have been investigated using this scaffold including tendon, ligament, skeletal muscle, and ventricular myocardium. Future work will be focused on further recapitulation of the native properties of the targeted tissues, and the temporal events conducive to tissue regeneration.

Acknowledgments This research was funded in part by NIH R01HL115282 (G.R.G., G.D.P.) and NSF IGERT DGE 1144804 (M.P.O., M.E.C., G.R.G). George D. Pins report grants from USAMRMC, and Raymond L. Page reports grants from DARPA and USAMRMC. 


\section{Compliance with Ethical Standards}

Conflict of Interest Megan P. O'Brien, Meagan E. Carnes, and Raymond L. Page declare that they have no conflict of interest.

George D. Pins and Glenn R. Gaudette disclose that they are cofounders and have equity interest in Vitathreads L.L.C., a company that has licensed intellectual property associated with fibrin microthreads, and they have several patents that are either issued or pending relevant to fibrin sutures and their production.

Human and Animal Rights and Informed Consent This article does not contain any studies with human or animal subjects performed by any of the authors.

Ethical approval All procedures performed in studies involving animals were in accordance with the ethical standards of the institution or practice at which the studies were conducted.

\section{References}

Papers of particular interest, published recently, have been highlighted as:

- Of importance

•. Of major importance

1. Atala A, Kasper FK, Mikos AG. Engineering complex tissues. Sci Transl Med. 2012;4:160rv12.

2. Grasman JM, Pumphrey LM, Dunphy M, et al. Static axial stretching enhances the mechanical properties and cellular responses of fibrin microthreads. Acta Biomater. 2014;10:4367-76.

3. Guyette JP, Fakharzadeh M, Burford EJ, et al. A novel suture-based method for efficient transplantation of stem cells. J Biomed Mater Res A. 2013;101:809-18. This is the first study to investigate the use of fibrin microthread-based sutures for stem cell delivery for cardiac applications.

4. Page RL, Malcuit C, Vilner L, et al. Restoration of skeletal muscle defects with adult human cells delivered on fibrin microthreads. Tissue Eng A. 2011;17:2629-40.

5. Altman GH, Diaz F, Jakuba C, et al. Silk-based biomaterials. Biomaterials. 2003;24:401-16.

6. Altman GH, Horan RL, Lu HH, et al. Silk matrix for tissue engineered anterior cruciate ligaments. Biomaterials. 2002;23: 4131-41.

7. Cornwell KG, Lei P, Andreadis ST, et al. Crosslinking of discrete self-assembled collagen threads: effects on mechanical strength and cell-matrix interactions. J Biomed Mater Res A. 2007;80A:362-71.

8. Gentleman E, Lay AN, Dickerson DA, et al. Mechanical characterization of collagen fibers and scaffolds for tissue engineering. Biomaterials. 2003;24:3805-13.

9. Funakoshi T, Majima T, Iwasaki N, et al. Application of tissue engineering techniques for rotator cuff regeneration using a chitosan-based hyaluronan hybrid fiber scaffold. Am J Sport Med. 2005;33:1193-201.

10. Funakoshi T, Majima T, Iwasaki N, et al. Novel chitosan-based hyaluronan hybrid polymer fibers as a scaffold in ligament tissue engineering. J Biomed Mater Res A. 2005;74A:338-46.

11. Irie T, Majima T, Sawaguchi N, et al. Biomechanical and histologic evaluation of tissue engineered ligaments using chitosan and hyaluronan hybrid polymer fibers: a rabbit medial collateral ligament reconstruction model. J Biomed Mater Res A. 2011;97A:111-7.

12. Baraniak PR, McDevitt TC. Stem cell paracrine actions and tissue regeneration. Regen Med. 2010;5:121-43.

13. Martins AM, Vunjak-Novakovic G, Reis RL. The current status of iPS cells in cardiac research and their potential for tissue engineering and regenerative medicine. Stem Cell Rev. 2014;10:177-90. This review focuses on the potential and associated challenges of using iPS cells for cardiac applications.

14. Vats A, Bielby RC, Tolley NS, et al. Stem cells. Lancet. 2005;366: 592-602.

15. Liu H, Fan H, Toh SL, et al. A comparison of rabbit mesenchymal stem cells and anterior cruciate ligament fibroblasts responses on combined silk scaffolds. Biomaterials. 2008;29:1443-53.

16. Proulx MK, Carey SP, Ditroia LM, et al. Fibrin microthreads support mesenchymal stem cell growth while maintaining differentiation potential. J Biomed Mater Res A. 2011;96:301-12.

17. Tao ZW, Favreau JT, Guyette JP, et al. Delivering stem cells to the healthy heart on biological sutures: effects on regional mechanical function. J Tissue Eng Regen Med. 2014. doi:10.1002/term.1904.

18. Grasman JM, Page RL, Dominko T, et al. Crosslinking strategies facilitate tunable structural properties of fibrin microthreads. Acta Biomater. 2012;8:4020-30.

19. Grasman JM, Do DM, Page RL, et al. Rapid release of growth factors regenerates force output in volumetric muscle loss injuries. Biomaterials. 2015;72:49-60. This paper describes functional regeneration achieved from fibrin microthread-mediated delivery of HGF in a murine model of VML.

20. Cornwell KG, Pins GD. Discrete crosslinked fibrin microthread scaffolds for tissue regeneration. J Biomed Mater Res A. 2007;82: 104-12

21. Cornwell KG, Pins GD. Enhanced proliferation and migration of fibroblasts on the surface of fibroblast growth factor-2-loaded fibrin microthreads. Tissue Eng A. 2010;16:3669-77.

22. Horan RL, Collette AL, Lee C, et al. Yarn design for functional tissue engineering. J Biomech. 2006;39:2232-40.

23. Chen J, Altman GH, Karageorgiou V, et al. Human bone marrow stromal cell and ligament fibroblast responses on RGD-modified silk fibers. J Biomed Mater Res A. 2003;67:559-70.

24. Dunn MGTA, Kato YP, Bechler JR, et al. Anterior cruciate ligament reconstruction using a composite collagenous prosthesis. A biomechanical and histologic study in rabbits. Am J Sports Med. 1992;5:507-15.

25. Zeugolis DI, Paul GR, Attenburrow G. Cross-linking of extruded collagen fibers-a biomimetic three-dimensional scaffold for tissue engineering applications. J Biomed Mater Res A. 2009;89:895908.

26. Pins GD, Christiansen DL, Patel R, et al. Self-assembly of collagen fibers. Influence of fibrillar alignment and decorin on mechanical properties. Biophys J. 1997;73:2164-72.

27. Zeugolis DI, Paul RG, Attenburrow G. The influence of a natural cross-linking agent (Myrica rubra) on the properties of extruded collagen fibres for tissue engineering applications. Mater Sci Eng C Mater. 2010;30:190-5.

28. Kato YP, Dunn MG, Zawadsky JP, et al. Regeneration of Achilles tendon with a collagen tendon prosthesis. Results of a one-year implantation study. J Bone Joint Surg Am. 1991;73:561-74.

29. Walters VI, Kwansa AL, Freeman JW. Design and analysis of braid-twist collagen scaffolds. Connect Tissue Res. 2012;53:25566.

30. Awad HA, Boivin GP, Dressler MR, et al. Repair of patellar tendon injuries using a cell-collagen composite. J Orthop Res. 2003;21: 420-31.

31. Matava MJ, and B. P. Boden. AOSSM initiates multi-center ACL revision study. Sports Medicine Update 2005; 5. 
32. Leong NL, Petrigliano FA, McAllister DR. Current tissue engineering strategies in anterior cruciate ligament reconstruction. J Biomed Mater Res A. 2014;102:1614-24. This review describes current strategies for applications in ACL tissue regeneration, including scaffold design, cell sources, growth factor delivery, and mechanical stimuli.

33. Silver FH. Biomaterials, medical devices, and tissue engineering: an integrated approach. London: Chapman \& Hall; 1994. p. 92-119.

34. Woo SL, Hollis JM, Adams DJ, et al. Tensile properties of the human femur-anterior cruciate ligament-tibia complex. The effects of specimen age and orientation. Am J Sports Med. 1991;19:217-25.

35. Kardestuncer T, McCarthy MB, Karageorgiou V, et al. RGDtethered silk substrate stimulates the differentiation of human tendon cells. Clin Orthop Relat Res. 2006;448:234-9.

36. Awad HA, Butler DL, Boivin GP, et al. Autologous mesenchymal stem cell-mediated repair of tendon. Tissue Eng. 1999;5:267-77.

37. Young R, Butler D, Weber W, et al. Use of mesenchymal stem cells in a collagen matrix for Achilles tendon repair. J Orthop Res. 1998;16:406-13.

38. Chen XQY, Wang LL, Yin Z, et al. Ligament regeneration using a knitted silk scaffold combined with collagen matrix. Biomaterials. 2008;29:3683-92.

39. Molloy T, Wang Y, Murrell G. The roles of growth factors in tendon and ligament healing. Sports Med. 2003;33:381-94.

40. Devore DI, Walters TJ, Christy RJ, et al. For combat wounded: extremity trauma therapies from the USAISR. Mil Med. 2011;176:660-3.

41. Rudnicki MA, Le Grand F, McKinnell I, et al. The molecular regulation of muscle stem cell function. Cold Spring Harb Symp Quant Biol. 2008;73:323-31.

42. De Coppi P, Bellini S, Conconi MT, et al. Myoblast-acellular skeletal muscle matrix constructs guarantee a long-term repair of experimental full-thickness abdominal wall defects. Tissue Eng. 2006;12: 1929-36.

43. Bianchi B, Copelli C, Ferrari S, et al. Free flaps: outcomes and complications in head and neck reconstructions. J Craniomaxillofac Surg. 2009;37:438-42.

44. Mase Jr VJ, Hsu JR, Wolf SE, et al. Clinical application of an acellular biologic scaffold for surgical repair of a large, traumatic quadriceps femoris muscle defect. Orthopedics. 2010;33:511.

45. Qazi TH, Mooney DJ, Pumberger M, et al. Biomaterials based strategies for skeletal muscle tissue engineering: existing technologies and future trends. Biomaterials. 2015;53:502-21.

46. Huard J, Li Y, Fu FH. Muscle injuries and repair: current trends in research. J Bone Joint Surg. 2002;84A:822-32.

47. Gilbert PM, Havenstrite KL, Magnusson KE, et al. Substrate elasticity regulates skeletal muscle stem cell self-renewal in culture. Science. 2010;329:1078-81.

48. Caiozzo VJ. Plasticity of skeletal muscle phenotype: mechanical consequences. Muscle Nerve. 2002;26:740-68.

49. Wolf MT, Dearth CL, Sonnenberg SB, et al. Naturally derived and synthetic scaffolds for skeletal muscle reconstruction. Adv Drug Deliv Rev. 2015;84:208-21. This review describes current natural and synthetic tissue engineering strategies for skeletal muscle regeneration.

50. Sicari BM, Dearth CL, Badylak SF. Tissue engineering and regenerative medicine approaches to enhance the functional response to skeletal muscle injury. Anat Rec. 2014;297:51-64.

51. Londono R, Badylak SF. Biologic scaffolds for regenerative medicine: mechanisms of in vivo remodeling. Ann Biomed Eng. 2015;43:577-92.

52. Grasman JM, Zayas MJ, Page RL, et al. Biomimetic scaffolds for regeneration of volumetric muscle loss in skeletal muscle injuries. Acta Biomater. 2015;25:2-15. This review describes the current biomimetic approaches to skeletal muscle regeneration.
53. Martinek V, Latterman C, Usas A, et al. Enhancement of tendonbone integration of anterior cruciate ligament grafts with bone morphogenetic protein-2 gene transfer: a histological and biomechanical study. J Bone Joint Surg Am. 2002;84-A:1123-31.

54. Gal-Levi R, Leshem Y, Aoki S, et al. Hepatocyte growth factor plays a dual role in regulating skeletal muscle satellite cell proliferation and differentiation. Biochim Biophys Acta. 1998;1402:39-51.

55. Tatsumi R, Anderson JE, Nevoret CJ, et al. HGF/SF is present in normal adult skeletal muscle and is capable of activating satellite cells. Dev Biol. 1998;194:114-28.

56. Grasman JM, Do DM, Page RL, et al. Rapid release of growth factors regenerates force output in VML injuries. Boston: TERMIS; 2015.

57. Deasy BM, Qu-Peterson Z, Greenberger JS, et al. Mechanisms of muscle stem cell expansion with cytokines. Stem Cells. 2002;20: $50-60$.

58. Rusnati M, Presta M. Interaction of angiogenic basic fibroblast growth factor with endothelial cell heparan sulfate proteoglycans. Biological implications in neovascularization. Int J Clin Lab Res. 1996;26:15-23.

59. Charge SB, Rudnicki MA. Cellular and molecular regulation of muscle regeneration. Physiol Rev. 2004;84:209-38.

60. Engert JC, Berglund EB, Rosenthal N. Proliferation precedes differentiation in IGF-I-stimulated myogenesis. J Cell Biol. 1996;135: $431-40$

61. Hawke TJ, Garry DJ. Myogenic satellite cells: physiology to molecular biology. J Appl Physiol. 2001;91:534-51.

62. Stoppel WL, Ghezzi CE, McNamara S, et al. Clinical applications of naturally derived biopolymer-based scaffolds for regenerative medicine. Ann Biomed Eng. 2015;43(3):657-80.

63. Mozaffarian D, Benjamin EJ, Go AS, et al. Heart disease and stroke statistics-2015 update: a report from the American Heart Association. Circulation. 2015;131:e29-322.

64. Frangogiannis NG, Smith CW, Entman ML. The inflammatory response in myocardial infarction. Cardiovasc Res. 2002;53:31-47.

65. Sutton MG, Sharpe N. Left ventricular remodeling after myocardial infarction: pathophysiology and therapy. Circulation. 2000;101: 2981-8.

66. Black 3rd LD, Meyers JD, Weinbaum JS, et al. Cell-induced alignment augments twitch force in fibrin gel-based engineered myocardium via gap junction modification. Tissue Eng A. 2009;15:3099108.

67. Sommer G, Haspinger D, Andra M, et al. Quantification of shear deformations and corresponding stresses in the biaxially tested human myocardium. Ann Biomed Eng. 2015;43:2334-48.

68. Kim DH, Lipke EA, Kim P, et al. Nanoscale cues regulate the structure and function of macroscopic cardiac tissue constructs. Proc Natl Acad Sci U S A. 2010;107:565-70.

69. Lee WN, Pernot M, Couade M, et al. Mapping myocardial fiber orientation using echocardiography-based shear wave imaging. IEEE Trans Med Imaging. 2012;31:554-62.

70. Coulombe KL, Bajpai VK, Andreadis ST, et al. Heart regeneration with engineered myocardial tissue. Annu Rev Biomed Eng. 2014;16:1-28.

71.• Radisic M, Christman KL. Materials science and tissue engineering: repairing the heart. Mayo Clin Proc. 2013;88:884-98. This review outlines the essential criteria necessary to produce a tissue engineered scaffold with the potential to promote tissue regeneration in cardiac applications.

72. Reis LA, Chiu LL, Feric N, et al. Biomaterials in myocardial tissue engineering. J Tissue Eng Regen Med. 2014. doi:10.1002/term. 1944.

73. Kraitchman DL, Tatsumi M, Gilson WD, et al. Dynamic imaging of allogeneic mesenchymal stem cells trafficking to myocardial infarction. Circulation. 2005;112:1451-61. 
74. Barbash IM, Chouraqui P, Baron J, et al. Systemic delivery of bone marrow-derived mesenchymal stem cells to the infarcted myocardium: feasibility, cell migration, and body distribution. Circulation. 2003;108:863-8.

75. Hou D, YoussefEA, Brinton TJ, et al. Radiolabeled cell distribution after intramyocardial, intracoronary, and interstitial retrograde coronary venous delivery: implications for current clinical trials. Circulation. 2005;112:I150-6.

76. Leor J, Gerecht S, Cohen S, et al. Human embryonic stem cell transplantation to repair the infarcted myocardium. Heart. 2007;93:1278-84.

77. Simpson D, Liu H, Fan TH, et al. A tissue engineering approach to progenitor cell delivery results in significant cell engraftment and improved myocardial remodeling. Stem Cells. 2007;25:2350-7.

78. Xiang Z, Liao R, Kelly MS, et al. Collagen-GAG scaffolds grafted onto myocardial infarcts in a rat model: a delivery vehicle for mesenchymal stem cells. Tissue Eng. 2006;12:2467-78.

79. Zimmermann WH, Melnychenko I, Eschenhagen T. Engineered heart tissue for regeneration of diseased hearts. Biomaterials. 2004;25:1639-47.

80. Laflamme MA, Chen KY, Naumova AV, et al. Cardiomyocytes derived from human embryonic stem cells in pro-survival factors enhance function of infarcted rat hearts. Nat Biotechnol. 2007;25: 1015-24.

81. Ogura N, Kawada M, Chang WJ, et al. Differentiation of the human mesenchymal stem cells derived from bone marrow and enhancement of cell attachment by fibronectin. J Oral Sci. 2004;46:207-13.
82. Menasche P. Cardiac cell therapy: lessons from clinical trials. J Mol Cell Cardiol. 2011;50:258-65.

83. Jakob P, Landmesser U. Current status of cell-based therapy for heart failure. Curr Heart Fail Rep. 2013;10:165-76.

84. Bhana B, Iyer RK, Chen WL, et al. Influence of substrate stiffness on the phenotype of heart cells. Biotechnol Bioeng. 2010;105: $1148-60$.

85. Vunjak-Novakovic G, Tandon N, Godier A, et al. Challenges in cardiac tissue engineering. Tissue Eng B Rev. 2010;16:169-87.

86. Zhang D, Shadrin IY, Lam J, et al. Tissue-engineered cardiac patch for advanced functional maturation of human ESC-derived cardiomyocytes. Biomaterials. 2013;34:5813-20.

87. El-Sherbiny IM, Yacoub MH. Hydrogel scaffolds for tissue engineering: progress and challenges. Glob Cardiol Sci Pract. 2013;2013:316-42.

88. Tandon V, Zhang B, Radisic M, et al. Generation of tissue constructs for cardiovascular regenerative medicine: from cell procurement to scaffold design. Biotechnol Adv. 2013;31:722-35.

89. O'Brien MP, Hansen KJ, Gaudette GR, et al. Fabrication of a fibrinbased myocardial layer for use in a modular cardiac patch. Tampa: Presented at the annual meeting of the Biomedical Engineering Society; 2015.

90. Vratsanos MA, O'Brien MP, Gaudette GR, et al. Modulating the mechanical properties of composite fibrin scaffolds. Tampa: Presented at the annual meeting of the Biomedical Engineering Society; 2015. 